\title{
Treatment of Wrinkles and Acne Scars Using the TriFractional, a Novel Fractional Radiofrequency Technology — Clinical and Histological Results
}

\author{
Alex Levenberg ${ }^{1}$, Andrea Gat ${ }^{2}$, Marie Christine Branchet ${ }^{3}$, Sylvie Boisnic ${ }^{3}$ \\ ${ }^{1}$ Physicians Center, Plastic Surgery Department, Tel Aviv, Israel; ${ }^{2}$ Dermatopathology Unit, Sourasky Medical Center, Tel Aviv, \\ Israel; ${ }^{3}$ GREDECO Research Group, Paris, France. \\ Email: leveplas@netvision.net.il
}

Received June $12^{\text {th }}, 2012$; revised July $15^{\text {th }}, 2012$; accepted July $31^{\text {st }}, 2012$

\begin{abstract}
Introduction: The need for cosmetic facial enhancement procedures with minimal down time and low risk has led to the development of methods for non-surgical skin rejuvenation. Various ablative lasers were developed, which remove the full skin surface in a controlled manner. However, the prolonged recovery and the significant risks prompted the development of fractional lasers which ablate the skin in a fractional manner, leaving untreated areas to improve healing process. In the past few years, fractional radiofrequency (RF) systems have been introduced that enable controlled skin resurfacing accompanied with dermal collagen remodeling. The new TriFractional technology from Pollogen ${ }^{\circledR}$ is designed to enable skin resurfacing and treatment of wrinkles using RF energy. The objective of the current research was to evaluate the safety and effectiveness of the novel TriFractional technology using the TriFractional applicator (A3F) for micro-ablative skin resurfacing and the treatment of wrinkles and acne scars. Methods: Healthy volunteers consented to undergo TriFractional treatments for various aesthetic indications. In addition, in-vivo and ex-vivo histological results showing immediate and long term effects of the TriFractional technology were obtained. A portion of the subjects underwent the complete TriLipo $\mathrm{Med}^{\mathrm{TM}}$ Procedure which includes 2 TriFractional treatments spaced one month apart, with 2 TriLipo RF + Dynamic Muscle Activation (DMA) face treatments two weeks after each TriFractional treatment. Results: Subjects experienced an improvement of wrinkles, skin texture and acne scars along with facial contouring. No significant adverse effects were detected post-treatment. Histology findings demonstrated immediate and long term TriFractional effects on both epidermal and dermal skin layers. Conclusions: TriFractional is a promising technology for skin resurfacing, treatment of wrinkles and improvement of additional aesthetic indications such as acne scars. The TriLipo $\mathrm{MED}^{\mathrm{TM}}$ procedure is a unique combination approach for total facial rejuvenation and contouring.
\end{abstract}

Keywords: Radiofrequency; TriFractional; Wrinkles; Acne Scars; Collagen

\section{Introduction}

The growing market demand for cosmetic facial enhancement and rejuvenation procedures with minimal down time and low risk, has led to the development of methods for non-surgical skin rejuvenation.

Laser skin resurfacing was first introduced in the mid 1990 's to precisely remove the upper layers of photo damaged, aged skin as an alternative to chemical peels. While enabling impressive clinical results, the first generation of laser resurfacing systems were associated with prolonged healing and high complication rates, particularly in darker skin patients [1,2]. These limitations resulted from the fact that the entire epidermis and papillary dermis were removed, often over large skin areas such as the entire face.

A new generation of laser resurfacing systems was introduced in 2003 with the development of a fractional laser (Reliant) $[3,4]$. Instead of treating an entire skin area, this laser treats a condensed matrix of small "islets" of tissue leaving intact skin in-between these islets. Healing is immediately initiated from these intact skin areas resulting in reduced downtime and reduced complications. Since the introduction of this first fractional laser system, a myriad of "fractional" laser systems, mostly based on $\mathrm{CO}_{2}$ and Erbium YAG laser technology, have been developed and cleared for marketing worldwide [5].

$\mathrm{RF}$ energy is a form of electromagnetic energy. When applied to tissue, rapidly oscillating electromagnetic fields cause movement of charged particles within the tissue 
and the resultant molecular motion generates heat. This source of heat has been extensively used in surgery for hemostasis and tissue ablation (electro-surgery). In the past decade RF has been widely applied in the field of aesthetics for various indications based on skin tightening generated by deep RF dermal heating with resultant collagen remodeling [6-8].

In the past several years, several fractional RF systems have been introduced that allow controlled fractional skin resurfacing, similar to the results achieved with various laser systems. In addition to the epidermal effect, fractional RF systems allow enhancement of the dermal layer thus contributing to a combined effect leading to an effective micro-ablative skin resurfacing and improved appearance of wrinkles, fine lines and acne scars.

The novel TriFractional approach using the new TriFractional applicator from Pollogen ${ }^{\circledR}$ is designed to enable fractional skin resurfacing and treatment of wrinkles via controlled epidermal micro-ablation and concomitant dermal remodeling with a long established, highly reliable, and cost-effective technology. Treatment with the new TriFractional applicator can be also done as part of a combination procedure, the TriLipo $\mathrm{MED}^{\mathrm{TM}}$ procedure, custom tailored to the patient, using additional technologies, TriLipo RF and TriLipo DMA, incorporated in the same device, the multi-application Maximus ${ }^{\mathrm{TM}}$ system.

The current research was intended to evaluate the safety and effectiveness of the novel TriFractional technology and applicator for skin resurfacing and treatment of wrinkles.

\section{Methods}

\subsection{TriFractional Technology}

The TriFractional applicator is indicated for skin resurfacing and the treatment of wrinkles by means of micro-ablation and coagulation. The TriFractional applicator is one of the modules on the Maximus system. A disposable treatment tip, containing a matrix of bi-polar electrode pins, is attached to the distal end of the applicator and is placed on the skin for treatment. TriFractional RF energy is delivered to the skin in a fractional manner via an array of multi-electrode pins.

Radiofrequency current is delivered sequentially between each of the pin electrodes and the large electrode which surrounds the pin matrix. Due to this design, relatively high RF current densities are formed in the tissue under each pin electrode, resulting in localized fractional treatment micro-wounds in the epidermis which are in direct contact with the electrodes while heat is delivered deeper into the dermis. This fractional manner of energy delivery leaves intact zones in between the targeted areas which serve as a reservoir of healthy cells to promote faster, more effective wound healing.

The TriFractional technology emits a "train" of three fractional sub-pulses spaced by very short pauses of Thermal Relaxation Time (TRT), in one treatment pulse. This modality enables to achieve an enhanced effect of deeper penetration and optimized efficacy, with less discomfort to the patient and a deeper gradual thermal effect.

Shortly after treatment there is moderate skin erythema and edema in the treated zone, which resolves within 1 3 days.

During the healing phase, small pin-point epidermal crusts appear at each micro-ablated spot. These crusts exfoliate naturally after a few days leading to mild epidermal resurfacing. In the dermis, treatment induces remodeling of dermal collagen (neocollagenesis) and of other matrix molecules, leading to dermal renewal.

\subsection{Clinical Methods}

A clinical evaluation was conducted treating healthy volunteers in order to assess the safety and efficacy of the TriFractional technology. The subjects were treated for various aesthetic reasons such as treatment of wrinkles, acne scars and un-even skin complexion.

The protocol included 2 or more (according to treated skin condition) treatments with the TriFractional applicator at four weeks interval.

In addition, selected subjects were treated using the TriLipo Med ${ }^{\mathrm{TM}}$ procedure which includes:

- Week 1: TriFractional

- Week 3: TriLipo focused RF + DMA

- Week 5: TriFractional

- Week 7: TriLipo focused RF + DMA

The addition of the TriLipo technology which combines TriLipo focused RF and DMA resulted in enhanced improvement of skin texture, toning and tightening. The TriLipo RF thermal effect stimulates collagen production while DMA is essential in improving lymphatic drainage and reducing edema.

Prior to treatment a medical history was taken to exclude any contraindication such as pregnancy, any implantable electronic device that could be disrupted by RF energy and any active dermatological or collagen-vascular disorder. Patients signed an informed consent form.

Topical anesthesia (Emla, AstraZeneca) was used on some of the subjects prior to the treatment according to the manufacturer instructions. The decision whether or not to use an anesthetic on treatment area was made by the physician based on size or sensitivity of the treatment area, treatment parameters and the subject's reported general tolerance.

The face was thoroughly cleaned and the treatment was performed only after the skin was completely dry. 
Subjects were provided with a "patient-controlled manual switch" which enabled them to stop the treatment should they experience significant discomfort. The TriFractional treatment parameters for each subject were defined according to the area being treated and the severity of the condition. During the first visit, the first few pulses were of low power level, in order to assess the immediate results. Once the initial skin response was assessed the physician decided on the power level according to subject's skin condition.

Subjects were asked to provide feedback regarding the pain level during treatment and were also questioned about the recovery process following each TriFractional treatment. Typically erythema and edema appeared and lasted for 1 - 3 days. A fractional eschar (matrix) pattern typically appeared 1 to 2 days post treatment and lasted 3 days to one week, depending on the skin characteristics and treatment strength. Subjects were instructed to use sun screen continuously to avoid risk of pigmentary changes.

\subsection{Histology}

To demonstrate the TriFractional effect on epidermal and dermal skin layers, preliminary tests were performed on porcine skin followed by comprehensive in-vivo and exvivo histological examinations.

\subsubsection{Porcine Skin}

Fresh porcine abdomen and ear skin samples were exposed to different TriFractional energy levels.

\subsubsection{In-Vivo Human Abdominal Skin}

One abdominal patient consented to a series of TriFractional pulses prior to her scheduled mini abdominoplasty. Pulses of the High power default settings $(160 \mathrm{msec})$ were performed at one week and one day before the surgery. Skin samples of treated and untreated control area were taken from this patient during her abdominoplasty procedure for histological analysis. Formalin fixed, paraffin embedded sections were prepared and stained with H\&E for pathological examination by Patho-Lab Diagnostics Ltd., Nes Ziona, Israel. Pathological examination was performed by Andrea Gat, M.D., Head of Dermatopathology Unit, Sourasky Medical Center, Tel Aviv, Israel.

\subsubsection{Ex-Vivo Human Skin Study}

The Gredeco ex vivo skin model for maintaining skin fragments (harvested from donors undergoing plastic surgery) in survival condition and artificially aging by Ultraviolet (UV) irradiation, was used as previously described by Boisnic and Branchet [7].
One treatment of the TriFractional applicator with a high power pulse $(150 \mathrm{msec})$ was performed.

- Histological modifications of the skin were analyzed at different intervals between Day 0 (D0) and Day 10 (D10), using H\&E staining.

- Immunohistochemical analysis of mitotic index of the epidermis was done on D2. An antibody Ki67 (Dako, clone MIB-1, marker cells in the M, S, G1, G2 phases of the cell cycle) was used. The immunodetection was made with an indirect technique of immunoperoxydase in 4 levels, amplified (kit DAKO) and stained in red by AEC (3-amino-9-ethylcarbazole).

The results were expressed in $\%$ of positive epithelial cells.

- Morphometrical analysis of collagen fibers was done at D10.

Serial sections of $4 \mu \mathrm{m}$ thickness were obtained and specifically stained with a picric acid solution containing $0.1 \%$ Sirius red. Collagen was analyzed by computerized morphometrical analysis. For a quantitative analysis of these macromolecules, a computerized image analysis of each section was made. The stained slides were examined using a microscope (Leitz) (magnification $\times 160$ ) connected with a camera unit (XC-75 CE type) and a microprocessor (Q520).

The surface of collagen bundles were measured in $\mu \mathrm{m}^{2}$ and the relative collagen content of the dermis was then expressed as a percentage.

For both the analyses of epidermal mitotic index and quantitative collagen content, samples with more than one pulse on the same area were included to amplify the reaction comparing to control samples.

\subsubsection{Statistical Analysis}

The statistical significance of changes recorded was determined using the Student's $t$-test when $\mathrm{p}<0.05$ was considered as statistically significant.

\section{Results}

\subsection{TriFractional Facial Treatments Results}

The results of the TriFractional treatments included reduction of fine wrinkles, improved appearance of acne scars, a general improvement of skin texture and scar tissue, skin brightening, skin tightening, improvement of skin irregularities and reduction in pore size. The subjects treated with the TriLipo Med procedure displayed an additional facial contouring effect especially visible on the jaw-line area.

No significant undesired effects were experienced by the subjects and they reported a tolerable pain level. All subjects expressed satisfaction with the clinical results.

Figures 1 - 3 demonstrate results of TriFractional treat- 
ments. Figure 1 shows impressive peri-oral wrinkles improvement after two TriFractional treatments.

Figure 2 demonstrates results following one TriFractional treatment for improvement of skin non-homogenous complexion post chemical peel, causing perioral hypopigmentation. Results demonstrated a substantial improvement after only one TriFractional treatment with a short recovery time. One week post treatment there were no scabs or other residual signs of the TriFractional treatment.

Figure 3 demonstrates impressive improvement of severe acne scars following TriFractional treatments.

\subsection{TriLipo MED Procedure Results}

Figures 4 - 6 demonstrate the effect of the TriLipo MED procedure for improvement of wrinkles and scars and for general facial contouring.
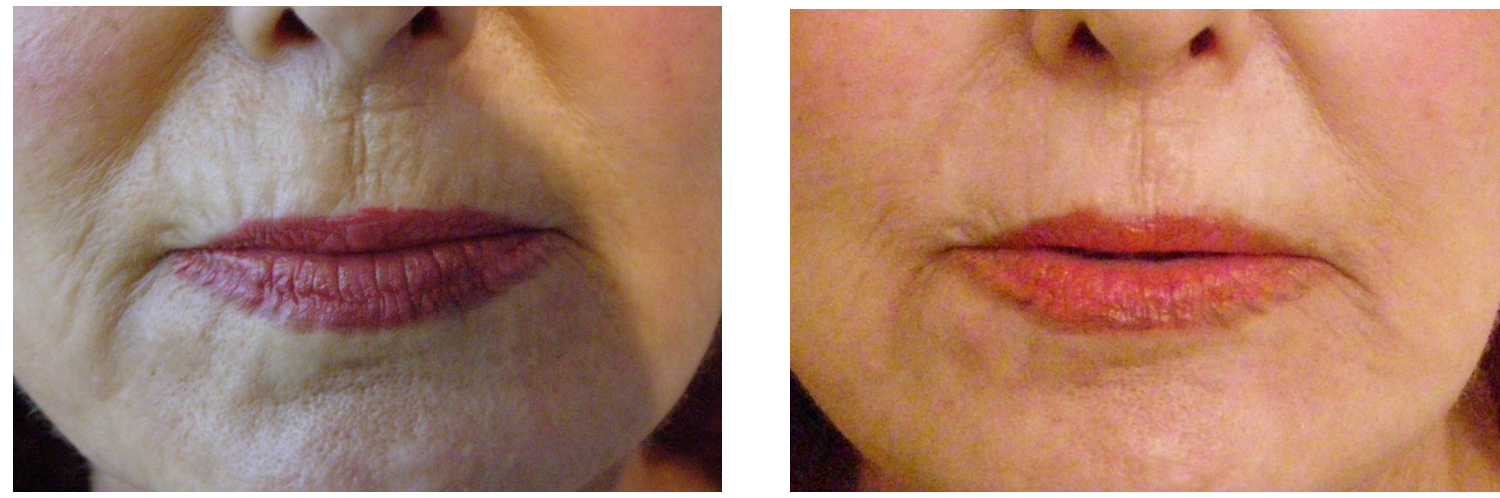

Figure 1. Peri-oral wrinkles improvement. Before (left) and after two TriFractional treatments (right).
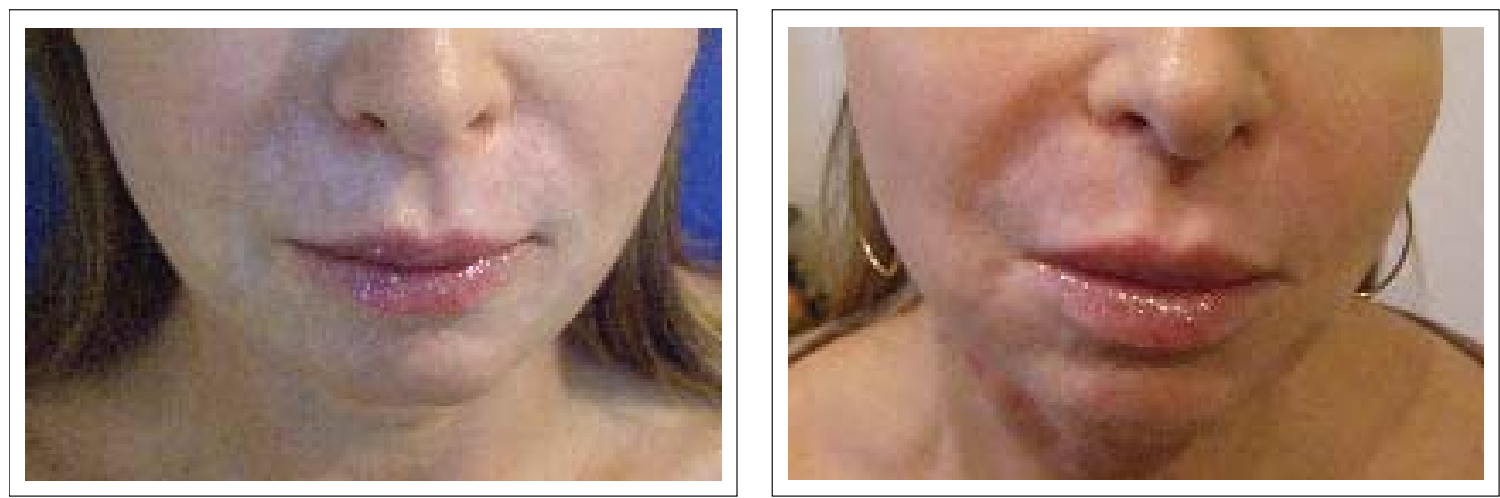

Figure 2. Skin revitalization. Before (left), after one week post one TriFractional treatment (right).
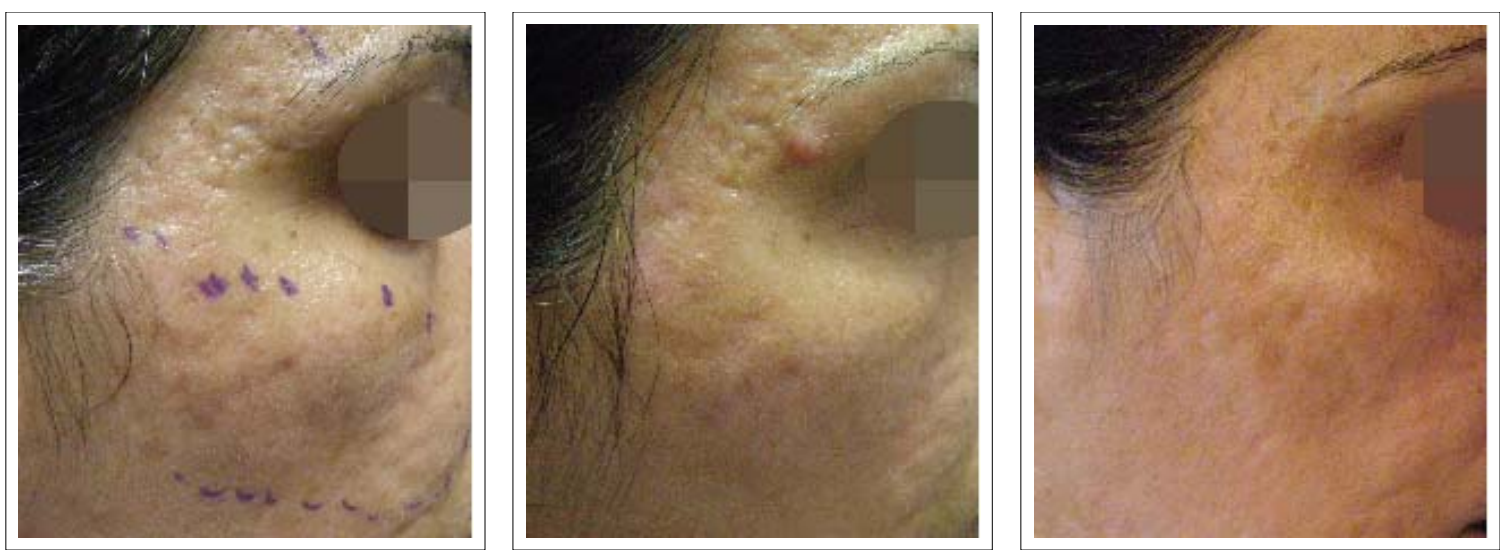

Figure 3. Treatment of acne scars. Baseline, multiple acne scars with uneven skin (left), 2 weeks post one TriFractional treatment (middle), 2 weeks post 3 TriFractional treatments (right). 

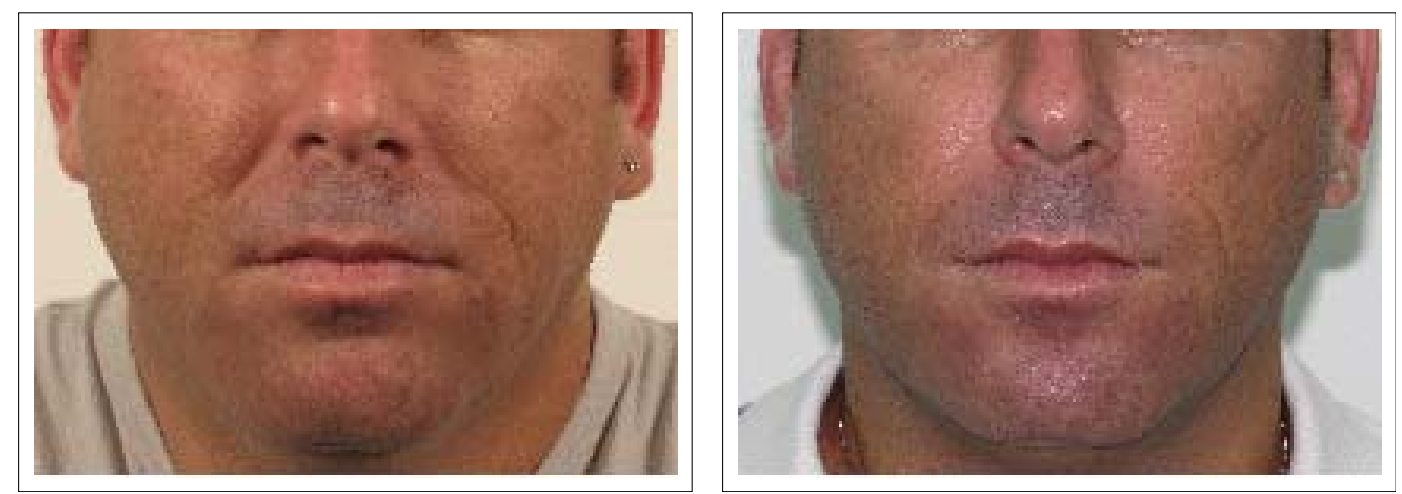

Figure 4. TriLipo MED Procedure - acne scars and skin texture improvement. Before (left) and after (right).
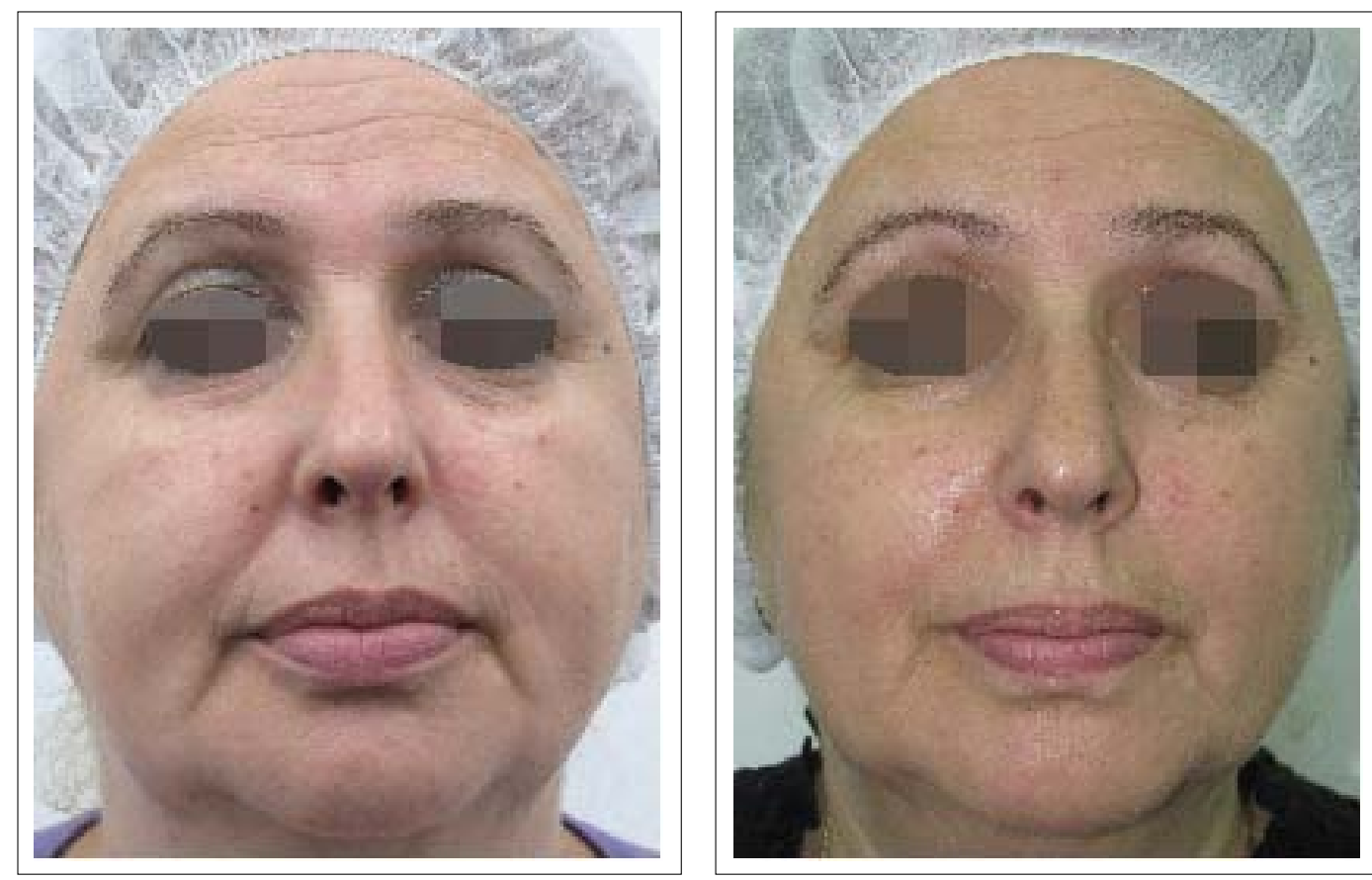

Figure 5. TriLipo MED Procedure showing the improvement of wrinkles and skin tightening. Before (left) and after (right).

The subjects underwent treatments with the TriFractional applicator on selected problematic areas, and additional complementary treatments with the TriLipo RF and DMA applicator on the entire face. Results indicate a significant facial contouring in lower cheeks and jaw line as well as improvement of wrinkles and acne scars. These results clearly demonstrate the additive value of the unique TriLipo MED procedure, combining TriFractional, TriLipo RF and TriLipo DMA technologies for facial skin rejuvenation and facial contouring.

\subsection{Histological Results}

\subsubsection{Porcine Skin Histologies for Preliminary Evaluation}

In all treated porcine skin samples there is thermal injury in the epidermis alone or up to and including the upper dermis, depending on the treatment parameters and thickness of skin fragment. The epidermal changes are characterized by elongation of nuclei, and by epidermal necrosis to various extents. The dermal changes are characterized by condensation in the dermal connective tissue.

Depth of injury is in direct correlation to the energy level used. In samples from abdomen skin treated with low power, epidermal injury was the least prominent and only the basal lower spinous epidermis layer was involved and the dermis was spared. The epidermal changes in this series were focal with skip area of normal epidermis in between the involved foci. All the other series showed both epidermal and dermal injury correlated with the level of energy in terms of depth and width. 

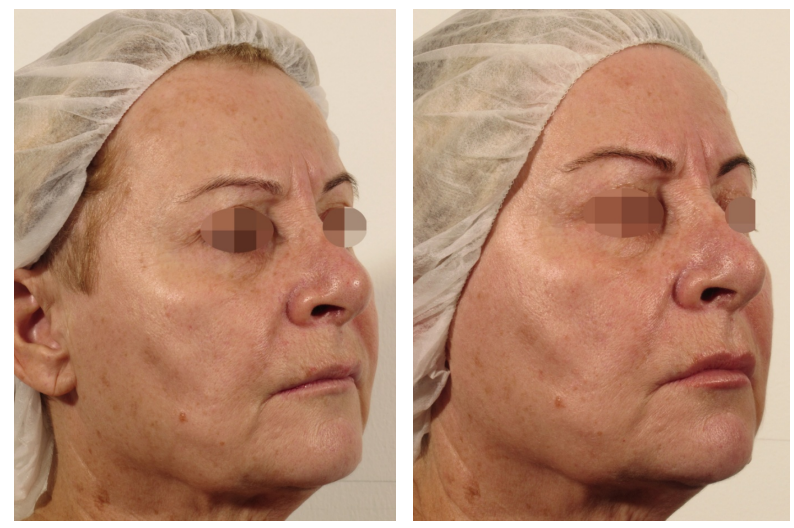

Figure 6. TriLipo MED Procedure showing skin tightening and improvement of lips appearance. Before (left) and after (right).

The ear skin samples are significantly more sensitive compared to abdomen skin samples, and even at low energy levels, there are areas of significant damage.

The histological results of the effect of varying power levels on the porcine skin emphasize the importance of careful selection of the treatment parameters. Figures 7 and $\mathbf{8}$ demonstrate the histological findings immediately following TriFractional treatment.

\subsubsection{In Vivo Human Abdominal Skin}

Samples taken one day after treatment showed epidermal necrosis in the involved area and an affect on the papillary as well as upper reticular dermal collagen areas. Skip areas are visible between affected areas. Neutrophilic infiltrate was present (Figure 9).

Samples taken 1 week after treatment show intact (regenerated) epidermis in foci covered with scale crust (sign of previous damage). Dermal collagen affected areas are demonstrated. Minimal lymphocytic infiltrate was present (Figure 10).

\subsubsection{Ex-Vivo Human Skin Study}

Histologies show epidermal modifications with a modified or necrotic appearance within 3 quarters of the epidermis or within all of the epidermis. In other cases, limited destruction of the epidermis was found with seconddary elimination of the necrosis shaped zone appearing as a squamous crust. In even rarer cases, alterations were very moderate, with condensed or vacuous spaces observed in the cytoplasm of the keratinocytes.

From D3 to D10 partial reparation was observed with the elimination of the crust (parakeratosis zone and necrotic debris), and regeneration of the basal cell layer under the modified epithelial zone (Figure 11).

\subsubsection{Evaluation of Mitotic Index of Epidermis}

The immunodetection realized just adjacent to the RF
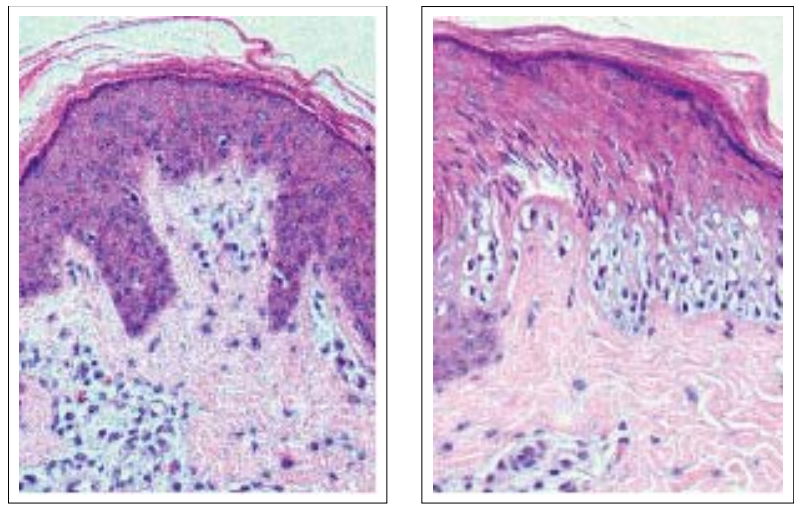

Figure 7. Porcine abdomen skin fragment treated with low energy (H\&E stain, ×200). Normal skin "with a skip area" between 2 electrodes (left). Epidermal thermal effect under electrode. Elongation of epidermal nuclei mainly in basal layer and lower spinous layer. No thermal effect observed in dermis (right).

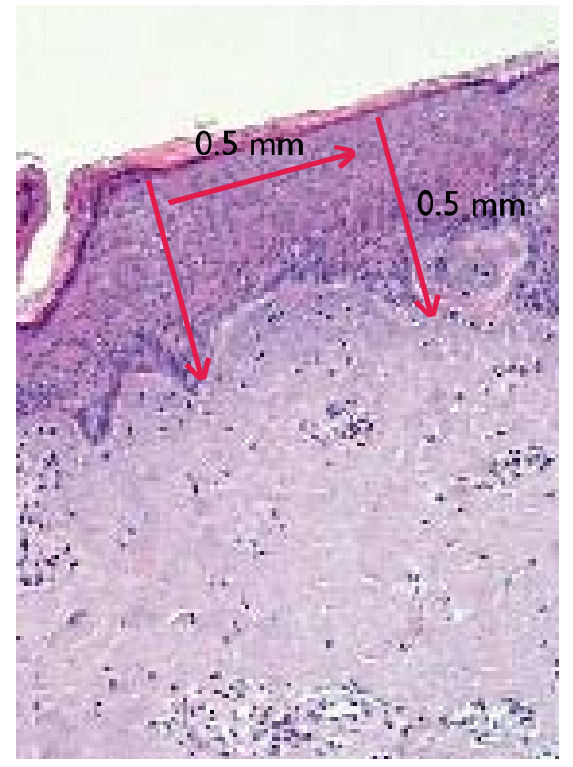

Figure 8. Porcine abdomen skin fragment treated with relatively high energy $(H \& E$ stain, $\times 100)$. Epidermis: areas of thermal damage with elongated epidermal nuclei at both sides (area under electrodes). In between at $\sim 0.5 \mathrm{~mm}$ width, effect is moderate. At bottom of skip area elongated nuclei are seen due to penetrated heat dispersion effect. Condensation of upper dermis is seen. Depth of injury $\sim 0.5 \mathrm{~mm}$.

treatment zones using antibody Ki67 revealed a statistically significant augmentation of positive epithelial cells (Figure 12). The percent of these mitotic cells rose from an average of $3.07 \% \pm 1.57 \%$ in experimentally aged skin to $8.9 \% \pm 3.03 \%$ in the skin treated by TriFractional RF $(p=0.0017)$. This represents an increase of nearly 3 times the amount of positive epithelial cells between experimentally aged skin and experimentally aged skin treated by fractional RF. 


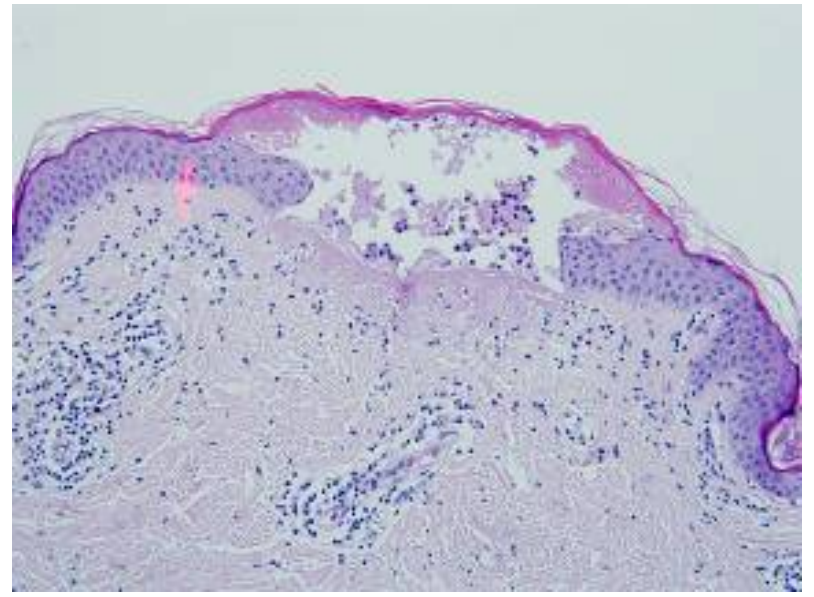

Figure 9. In-vivo abdominal skin histology 1 day after treatment $(H \& E$ stain, $\times 200)$. Epidermal damage covered by crust, dermal effect and neutrophilic infiltrate are demonstrated.


Figure 11. Ex-vivo UV aged skin histology at D3 (up) and D10 (bottom) in survival medium (H\&E stain magnification $\times 400: 1$ $\mathrm{cm}=19 \mu \mathrm{m})$. Left: untreated. Right: treated with TriFractional RF pulse. D3 treated sample demonstrate epidermal scarring with the formation of a new epidermis while old epidermis is eliminating. Condensation of upper dermal collagen fibers is observed. D10 treated samples demonstrate moderate alteration of the epidermis and dermis. 

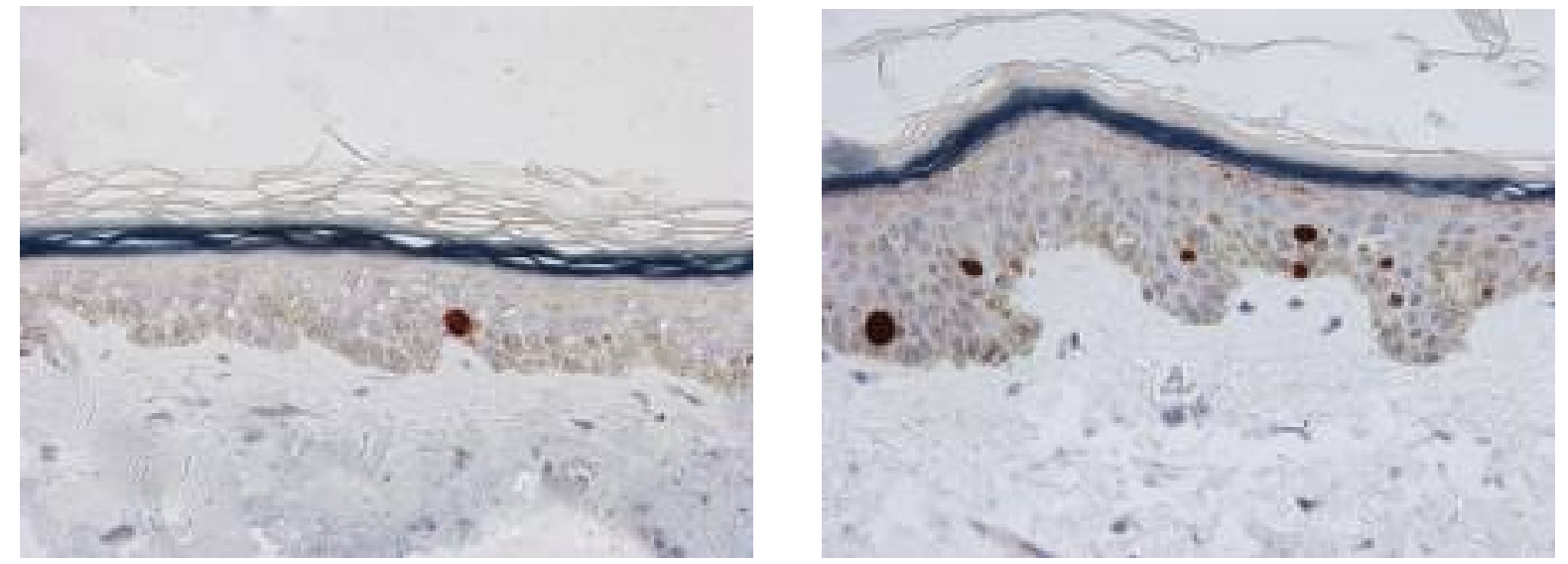

Figure 12. Ex-vivo UV aged skin histology at D2 in survival medium. Immunohistochemical analysis of mitotic cells in the epidermis $(\mathrm{Ki67}, \times 400: 1 \mathrm{~cm}=19 \mu \mathrm{m})$. Left: untreated. Right: treated with TriFractional RF pulse. Increase of mitotic cells in the zone adjacent to the fractional RF treatment area is demonstrated.

\subsubsection{Histological Quantification of Dermal Collagen by Computerized Image Analysis}

Morphometry of collagen revealed a statistically significant augmentation from $62.7 \% \pm 1.75 \%$ average collagen for experimentally aged skin to $72.5 \% \pm 4.07 \%$ collagen for skin treated by fractional RF $(p=0.00019)$. This corresponds to $15.5 \%$ increase of collagen content (Figure 13).

\section{Discussion and Conclusions}

During the past years, the fractional treatment concept became trendy as it offers a controlled skin treatment of specific tiny areas with enhanced healing through the surrounding untreated areas. Extensive attempts have been made to investigate the clinical and histopathologycal effects of fractional energy sources on dermal and epidermal structures.

Several fractional RF systems have recently been cleared for marketing based on published clinical results with these RF fractional systems [9-11].

Hruza et al. [9] evaluated the effect of a fractional RF applicator (Matrix ${ }^{\text {TM }}$ RF by Syneron) for skin rejuvenation and wrinkle reduction. Their histological findings, immediately post-treatment, revealed demarcated zones of ablation/coagulation/necrosis and subnecrosis up to a depth of 450 micron. Higher energy levels generated deeper effects. Subjects undergoing facial treatment had minimal pain, no permanent side effects, or significant downtime. The investigators' assessment of improvement in skin texture was greater than $40 \%$ for approximately $50 \%$ of subjects. Higher energy levels and lower coverage rates produced better aesthetic results along with less pain. They concluded that RF fractional skin resurfacing results in a safe, tolerable and effective improvement in skin texture and reduction of wrinkles. The depth of tis- sue ablation, coagulation and necrosis and the relative proportions of these phenomena were found to be controllable and could be modulated to optimize treatment of variable dermatologic conditions.

Another study [10] further evaluating the effect of the eMatrix $^{\mathrm{TM}}$ system by Syneron, confirmed that the treatment improved skin appearance while addressing some of the limitations of ablative resurfacing and non ablative skin rejuvenation. Authors reported that unlike fractional ablative laser treatments, which mostly affect the epidermis, the sublative technique has less impact on the epidermis and most of the effect is in the dermis. They concluded that healing is rapid and downtime is minimal thus treatment is appropriate for all skin types and is an effective alternative for patients with darker skin.

The current study demonstrates the safety and efficacy of the novel fractional RF technology, the TriFractional. The evaluation provides clinical and histological evidence supporting the TriFractional being a promising technology for skin rejuvenation, treatment of wrinkles and acne scars and for general improvement of skin texture, with minimal down time and minimal discomfort to the patient. Histological results confirmed the TriFractional impact on epidermal renewal and dermal regeneration and demonstrated the biological mechanism of the effect. Immediate effect as well as effect following a few days was demonstrated in various histological models, while exvivo results support the in-vivo findings. Combining the TriFractional treatment with intermediate TriLipo RF and TriLipo DMA using the TriLipo MED Procedure, enhances facial toning and enables a holistic approach to facial rejuvenation and enhancement.

In summary, the fractional mode of the treatment enables a faster, more effective healing process, eliminating patient downtime resulting in a safe, tolerable, effective skin rejuvenation. 

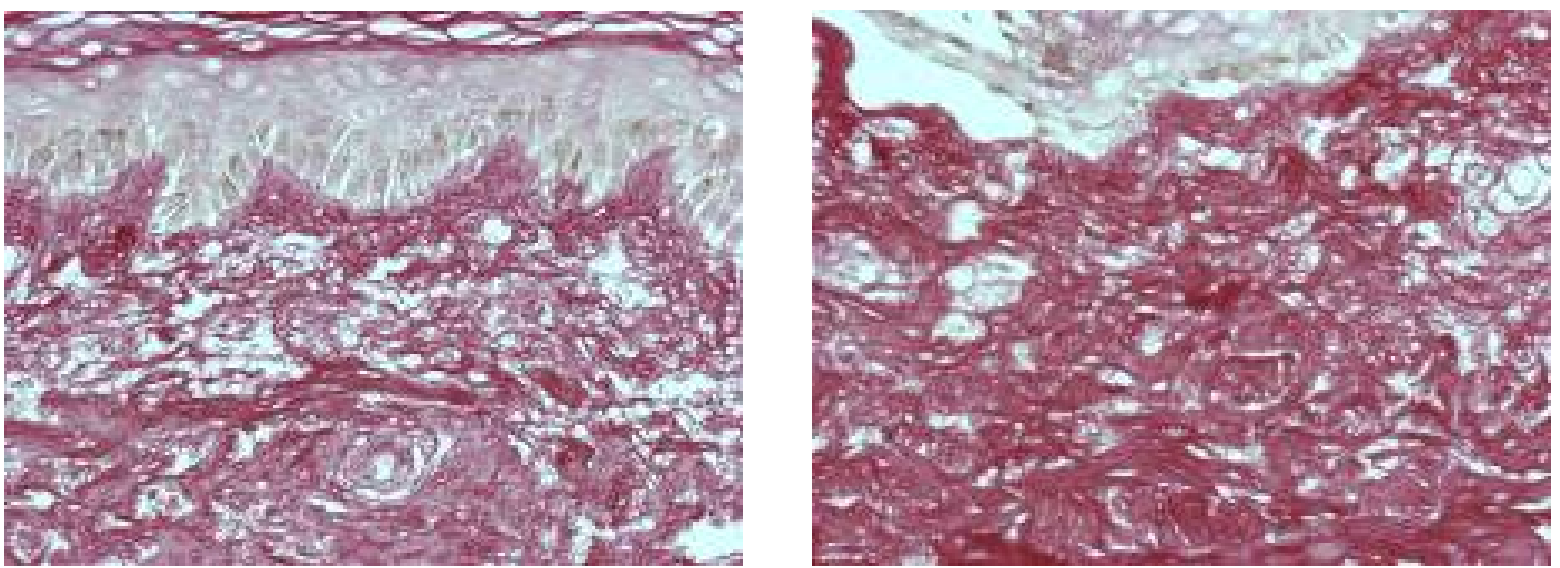

Figure 13. Ex-vivo UV aged skin histology at D10 in survival medium. Histological analysis of dermal collagen stained by sirius red $(\times 400: 1 \mathrm{~cm}=19 \mu \mathrm{m})$. Left: untreated. Right: treated with TriFractional RF pulse. Increase of collagen fibers in the dermis under the fractional RF treatment zone is demonstrated.

\section{Disclosures}

Dr. Levenberg acts as a medical director for Pollogen. The other authors received from Pollogen research grants for the corresponding study projects.

\section{REFERENCES}

[1] C. A. Nanni and T. S. Alster, "Complications of $\mathrm{CO}_{2}$ Laser Resurfacing: An Evaluation of 500 Patients," Dermatologic Surgery, Vol. 24, No. 3, 1998, pp. 315-320.

[2] E. L. Tanzi and T. S. Alster, "Side Effects and Complications of Variable Pulsed Erbium: YAG Laser Skin Resurfacing: Extended Experience with 50 Patients," Plastic and Reconstructive Surgery, Vol. 111, No. 4, 2003, pp. 1524-1529. doi:10.1097/01.PRS.0000049647.65948.50

[3] D. Manstein, G. S. Herron, R. K. Sink, et al., "Fractional Photothermolysis: A New Concept for Cutaneous Remodeling Using Microscopic Patterns of Thermal Injury," Lasers in Surgery and Medicine, Vol. 34, No. 5, 2004, pp. 426-438. doi:10.1002/1sm.20048

[4] H. J. Laubach, Z. Tannous, R. R. Anderson and D. Manstein, "Skin Responses to Fractional Photothermolysis," Lasers in Surgery and Medicine, Vol. 38, No. 2, 2006, pp. 142-149. doi:10.1002/lsm.20254

[5] E. P. Tierney, R. F. Eisen and C. W. Hanke, "Fractionated $\mathrm{CO}_{2}$ Laser Skin Rejuvenation," Dermatology and Therapy, Vol. 24, No. 1, 2011, pp. 41-53. doi:10.1111/j.1529-8019.2010.01377.x

[6] H. Kaplan and A. Gat, "Clinical and Histopathological Results Following TriPollar Radiofrequency Skin Treatments," Journal of Cosmetic and Laser Therapy, Vol. 11, No. 2, 2009, pp. 78-84.

[7] S. Boisnic and M. C. Branchet, "Ex Vivo Human Skin Evaluation of Localized Fat Reduction and Anti-Aging Effect by TriPollar Radio Frequency Treatments," Journal of Cosmetic and Laser Therapy, Vol. 12, No. 1, 2010, pp. 25-31. doi:10.3109/14764170903376232

[8] A. Levenberg, "Clinical Experience with a TriPollar Radiofrequency System for Facial and Body Aesthetic Treatments," European Journal of Dermatology, Vol. 20, No. 5, 2010, pp. 615-619.

[9] G. Hruza, A. F. Taub, S. L. Collier and S. R. Mulholland, "Skin Rejuvenation and Wrinkle Reduction Using a Fractional Radiofrequency System," Journal of Drugs in Dermatology, Vol. 8, No. 3, 2009, pp. 259-265.

[10] L. Brightman, M. P. Goldman and A. F. Taub, "Sublative Rejuvenation: Experience with a New Fractional Radiofrequency System for Skin Rejuvenation and Repair," Journal of Drugs in Dermatology, Vol. 8, No. 11, 2009, pp. 9-13.

[11] N. S. Sadick, M. Sato, D. Palmisano, et al., "In Vivo Animal Histology and Clinical Evaluation of Multisource Fractional Radiofrequency Skin Resurfacing (FSR) Applicator," Journal of Cosmetic and Laser Therapy, Vol. 13, No. 5, 2011, pp. 204-209. 